\title{
Numerical simulations of mass loading in the solar wind interaction with Venus
}

\author{
K. Murawski ${ }^{1}$ and R. S. Steinolfson \\ Department of Space Science, Southwest Research Institute, San Antonio, Texas
}

\begin{abstract}
Numerical simulations are performed in the framework of nonlinear two - dimensional magnetohydrodynamics to investigate the influence of mass loading on the solar wind interaction with Venus. The principal physical features of the interaction of the solar wind with the atmosphere of Venus are presented. The formation of the bow shock, the magnetic barrier, and the magnetotail are some typical features of the interaction. The deceleration of the solar wind due to the mass loading near Venus is an additional feature. The effect of the mass loading is to push the shock farther outward from the planet. The influence of different values of the magnetic field strength on plasma evolution is considered.
\end{abstract}

\section{Introduction}

Data from the Pioneer Venus Orbiter (PVO), as well as data from Venera 9 and 10 and other sources, have contributed to a growing understanding of the interaction of the solar wind with an unmagnetized planet such as Venus. The general picture derived from the available data is that, for typical solar wind conditions, an electrically conducting ionosphere deflects the supersonic and superalfvénic solar wind around the planet. A bow shock forms upstream of the ionosphere and serves to slow and also assists in deflecting the solar wind. The size of the bow shock depends on the sonic and alfvénic Mach numbers and on the shape and the size of the effective obstacle, whereas asymmetries in the shock shape are determined by the direction of the interplanetary magnetic field (IMF) [Khurana and Kivelson, 1994]. Zhang et al. [1990] have proven that at Venus the effective obstacle undergoes a systematic size change of $0.13 R_{v}$ (where $R_{v}$ is the radius of Venus) during a solar cycle. The boundary separating the shocked and magnetized solar wind plasma from the thermal ionosphere plasma is referred to as the ionopause, and the region between the bow shock and the ionosphere is referred to as the magnetosheath. The inner portion of the magnetosheath contains a region of enhanced magnetic pressure referred to as the magnetic barrier [e.g., Luhmann, 1986]. There are physical processes involved in these relatively large-scale interaction regions as well as in the magnetic tail that the present work has application to.

\footnotetext{
${ }^{1}$ Also at Faculty of Mechanics, Politechnic of Lublin, Lublin, Poland.
}

Copyright 1996 by the American Geophysical Union.

Paper number 95JA002433.

0148-0227/96/95JA-02433\$05.00
Zhang et al. [1991] have used PVO data to show that the convected field gasdynamic model of Spreiter et al. [1966] predicts the correct bow shock location if the magnetic barrier is taken as the obstacle around which the plasma is deflected. They also found that the magnetic barrier is strongest and thinnest (about 200 $\mathrm{km}$ thick) at the subsolar point and becomes weaker and thicker at the terminator. The magnetic barrier is responsible for transferring most of the solar wind dynamic pressure to the ionosphere through the enhanced magnetic pressure.

Observations at Venus have revealed that a magnetic tail forms as a consequence of the solar wind interaction with the Venusian ionosphere. The portions of IMF lines passing near the surface of Venus are slowed due to the interaction with newly ionized atmospheric neutrals. The two ends of the magnetic ropes continue to be pulled downstream by the solar wind [e.g., Spreiter and Stahara, 1980]. Slavin et al. [1989] have estimated that the magnetotail extends to between 50 to $150 R_{v}$ from the center of the planet for a typical solar wind flow with the Alfven speed $V_{A}=60 \mathrm{~km} / \mathrm{s}$ and the Alfvén Mach number $M_{A}=7.2$.

A study by Nagy et al. [1981] showed that Venus has a dayside neutral exosphere dominated by oxygen at altitudes above $\sim 400 \mathrm{~km}$ calculated from the planetary surface. The exospheric oxygen together with other particles present can be ionized by solar radiation and charge exchange. Newly ionized particles are electromagnetically coupled to the shocked solar plasma of the magnetosheath. As a consequence, near the ionopause the plasma becomes mass loaded due to interactions with ions, particularly $\mathrm{O}^{+}$[e.g., Luhmann et al., 1991]. Model computations by Gombosi et al., [1980] using input from PVO ionosheath and neutral atmosphere profiles indicate that approximately $10 \%$ by number of the solar wind protons may undergo charge exchange with neutrals at ionopause altitudes. As a result of the mass loading (ML), the plasma is slowed and compressed. When the plasma then flows around the planet into the 
magnetosheath, momentum conservation requires an increase in flow speed into the magnetotail.

It has been noted by Luhmann [1986] that the above general behavior tends to prevail providing the thermal pressure in the upper ionosphere is sufficiently larger than the solar wind pressure and the pressure balance occurs where the ionospheric plasma is collisionless. As the solar wind pressure increases, relative to that of the ionospheric plasma, the magnetic barrier eventually breaks down and the height of the dayside ionosphere decreases. When the ionopause is too close to the planet, the transterminator transport of ionospheric plasma ceases, thereby leading to a nightside phenomenon known as the disappearing of the ionosphere [Cravens et al., 1982].

Cloutier et al. [1987] have noted that ML processes at Venus should be asymmetric since the solar wind electric field depends on the relative directions of the flow velocity and the IMF. Phillips et al. [1987] have claimed that the flow and field configuration of the magnetosheath plasma, together with the large gyroradius of the pickup ions, cause ML to occur preferentially on one side of the magnetosheath. More efficient pickup of newly created ions should occur over the hemisphere that produces an electric field directed outward, away from the ionosphere, Luhmann et al. [1991] and Phillips et al. [1986] have presented observations indicating an increase in the magnetic field intensity and current over the hemisphere where the electric field is directed outward. The enhanced solar UV during solar maximum increases the ionization rate of neutrals, which moves the bow shock out from the planet both in the equatorial plane and at the terminator [Zhang et al., 1990]. Similarly, Alexander and Russell [1985] have shown that the position of the bow shock at the terminator depends on solar activity. This dependence may be indirect evidence of ML since, when the solar EUV is high, more mass may be added to the shocked solar wind, thereby forcing the bow shock to recede from the planet. Linker et al. [1989] have revealed that ML can increase or decrease the plasma temperature, depending on the value of the sonic Mach number $M_{S}$. When $\gamma=5 / 3$, $M_{S}>\sqrt{9 / 5}$ is required for heating to occur. Numerical simulations performed show that the effects of ML on plasma temperature and velocity are most pronounced in a wake region. However, this theory was developed for the case of Io's atmosphere, which is described by MHD equations with the source terms in the mass continuity, Euler, and energy equations. In the case of Io, the magnetic field lines are in the north-south direction and are perpendicular to the horizontal flow.

Recent computer simulations of the three-dimensional global interaction between the solar wind and unmagnetized bodies have proven to be extremely useful for improving our understanding of the associated largescale processes [e.g., Wu, 1992; Tanaka, 1993; Cable and Steinolfson, 1995; Gombosi et al. 1994; McGary and Pontius, 1994]. Venus is of particular interest since the planet does not have a significant intrinsic magnetic field, and the interaction of the solar wind with the Venusian ionosphere involves fundamentally differ- ent physical processes than occur at magnetized Earth. In addition, a large quantity of relevant observations are now available for Venus. When interpreted in association with the simulated results, this data set provides a test of the physical processes included in the model.

Single-fluid MHD simulations of the global interaction of the solar wind with Venus without consideration of the ionosphere have been performed by several investigators. $W u[1992]$ limited his study to the dayside. Tanaka [1993] included the nightside as well but only considered magnetic field orientations parallel and perpendicular to the solar wind flow and was in a parameter regime not directly applicable to average solar wind conditions at Venus. Cable and Steinolfson [1995] carried out simulations for typical observed solar wind conditions at Venus and for an IMF orientation near the Parker spiral. McGary and Pontius [1994] studied the effects of ML, but they considered only the dayside. Moreover, they discussed the case of a cylinder with $\partial / \partial z=0$ and $B$ along the cylinder axis ( $z$ - axis) and the perpendicular flow, namely, $\mathbf{V} \perp \mathbf{B}$.

Our purpose is to extend the model of McGary and Pontius to study the nightside and to discuss various strengths of the magnetic field. We also considered a more realistic spherical model.

The paper is organized as follows. The physical model used in the present study is described in the next section. Section 3 describes the numerical model which is used in the present studies. We present and discuss our detailed results in section 4 . The paper concludes with a short summary.

\section{Physical Model}

We solve the following form of the compressible MHD equations as an initial value problem:

$$
\begin{gathered}
\frac{\partial \rho}{\partial t}+\nabla \cdot(\rho \mathbf{V})=q_{0} \exp \left[-\left(h-h_{0}\right) / H_{0}\right] \\
\frac{\partial(\rho \mathbf{V})}{\partial t}+\nabla \cdot[(\rho \mathbf{V}) \mathbf{V}]=-\nabla p+\frac{1}{4 \pi}(\nabla \times \mathbf{B}) \times \mathbf{B} \\
\frac{\partial \mathbf{B}}{\partial t}=\nabla \times(\mathbf{V} \times \mathbf{B}) \\
\frac{\partial}{\partial t}\left(\frac{\rho V^{2}}{2}+\frac{p}{\gamma-1}+\frac{B^{2}}{8 \pi}\right)+\nabla \cdot\left\{\left(\frac{\rho V^{2}}{2}+\frac{\gamma}{\gamma-1} p\right) \mathbf{V}\right. \\
\left.+\frac{1}{4 \pi}[\mathbf{B} \times(\mathbf{V} \times \mathbf{B})]\right\}=0 \\
\nabla \cdot \mathbf{B}=0 .
\end{gathered}
$$

Here $\rho$ is the plasma density. $\mathbf{V}$ is the velocity. $\mathbf{B}$ is the magnetic field. $p$ is the plasma pressure, and $q_{0}$ is the production rate at a reference altitude $h_{0} . H_{0}$ is the scale height of the hot neutral oxygen population, and the ratio of specific heats is $\gamma=5 / 3$. 
In the present first-order approximation, the newly created photoions are considered as cold $(T=0)$ and motionless $(\mathbf{V}=0)$ particles. A more rigorous approach would be to add corresponding source terms to the Euler (2) and energy (4) equations [e.g., Gombosi et al., 1994]. However, it was shown by Biermann et al. [1967] that the major effect is that associated with the mass continuity equation and that contributions to the momentum and energy density are small. Therefore in these preliminary calculations source terms in the momentum and energy equations are neglected. The ML term is given by the right-hand side of the mass continuity equation (1). Oxygen photoionization is considered to be the only mass source with an altitude distribution described by the exponential function. This production rate is based on PVO observations for solar maximum, where the hot neutral oxygen atmosphere had a scale height $H_{0}=400 \mathrm{~km}$ in the subsolar region and a reference production rate of $q_{0}=3 \times 10^{5} \mathrm{~cm}^{-3} \mathrm{~s}^{-1}$ at altitude $h_{0}=400 \mathrm{~km}$ [Belotserkovskii et al., 1987]. As numerical simulations, which were made for various mass addition rates, revealed that the boundary layer develops when oxygen ion production rate is $q_{0}=3 \times 10^{5} \mathrm{~cm}^{-3} \mathrm{~s}^{-1}$ [McGary, 1993; McGary and Pontius, 1994], we carried on our simulations for this value of $q_{0}$.

\section{Numerical Model}

These numerical calculations were performed with HEMIS3D, a three - dimensional ideal MHD code. The code utilizes the modified Lax-Wendroff scheme developed by Rubin and Burstein [1967], which yields stable solutions by adding numerical diffusion to the scheme. The dissipative terms (kept as small as possible) are included to help damp out short-wavelength ripples generated by numerical dispersion and numerically induced reflections from the simulation boundaries, while leaving the longer-wavelength phenomena minimally affected. The equations are solved in all three spatial dimensions of a spherical $(r, \theta, \phi)$ coordinate system in which the $\theta=0$ axis is directed toward the Sun or into the solar wind flow. The generally small deviations of the solar wind flow from the radial direction (with respect to the Sun) are neglected so the solar wind flow impacting the Venusian ionosphere can be assumed parallel to the Sun-Venus line $(\theta=0)$. The spherical coordinate system is centered on the planet so $r=R_{v}$ represents the planetary surface.

Although a grid defined in spherical coordinates introduces some computational complexity, it has a number of advantages for studying flow past planetary obstacles such as Venus. One obvious advantage is that the boundary at the planetary surface occurs at a fixed value of the radial coordinate, which avoids the complications of interpolating in a Cartesian coordinate system. Another distinct advantage is that as the center of Venus is located at the center of the spherical coordinate system, high resolution near Venus is obtained without the expense of high resolution everywhere in the simulation. The code is constructed in such a man- ner that it can be applied to a single $r, \theta$ plane when the phenomenon being studied is cylindrically symmetric about the poles at $\theta=0$ and $\theta=180^{\circ}$, as is the case when the magnetic field is parallel to the solar wind
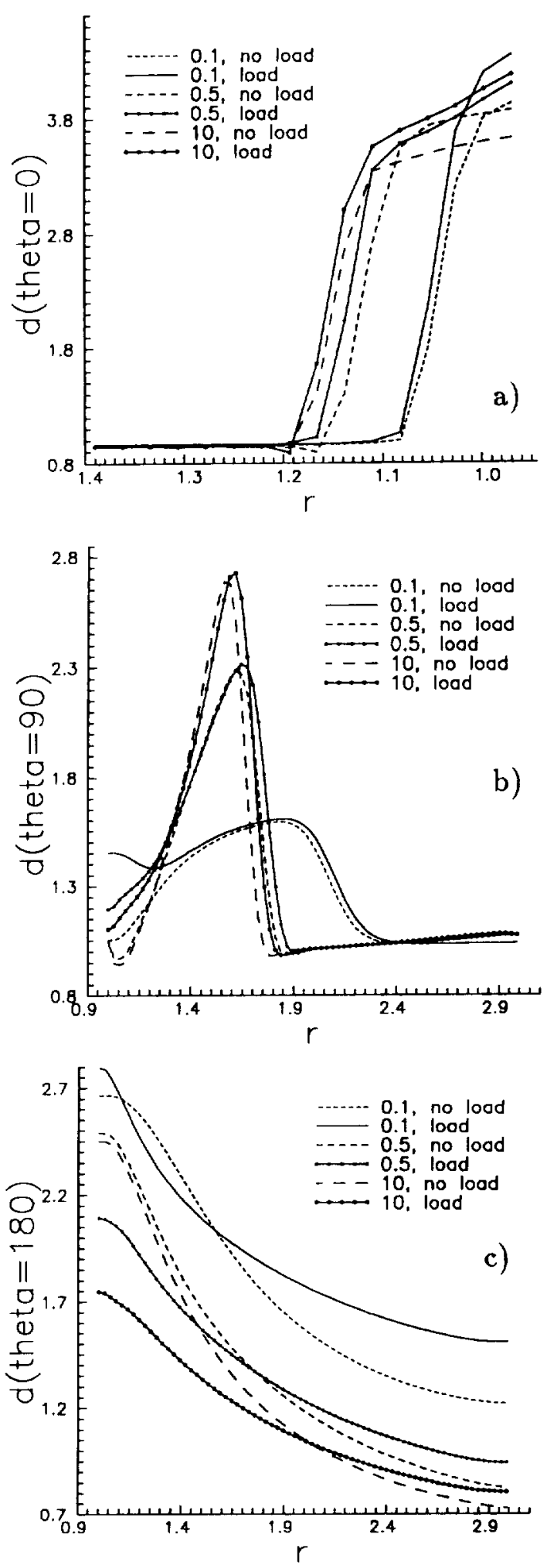

Figure 1. Plots of the normalized mass density profiles along $\theta$ $=$ const lines for $\beta=0.1, \beta=0.5, \beta=10$ and for the NL and ML simulations. (a) the Sun-Venus $(\theta=0)$ line, (b) the terminator $\left(\theta=90^{\circ}\right)$ line, and (c) the $\theta=180^{\circ}$ line. Mass loading shifts the bow shock farther away from Venus. 
flow. In this case, the simulation code uses a $73 \times 74$ grid ( $r, \theta$ grid dimensions), which corresponds to $\Delta \theta=2.5$, with a radial extent of the simulations of $3 R_{V}$. The largest $\Delta r=0.083 R_{V}$. We have checked that the results for larger extend of the numerical boundaries were
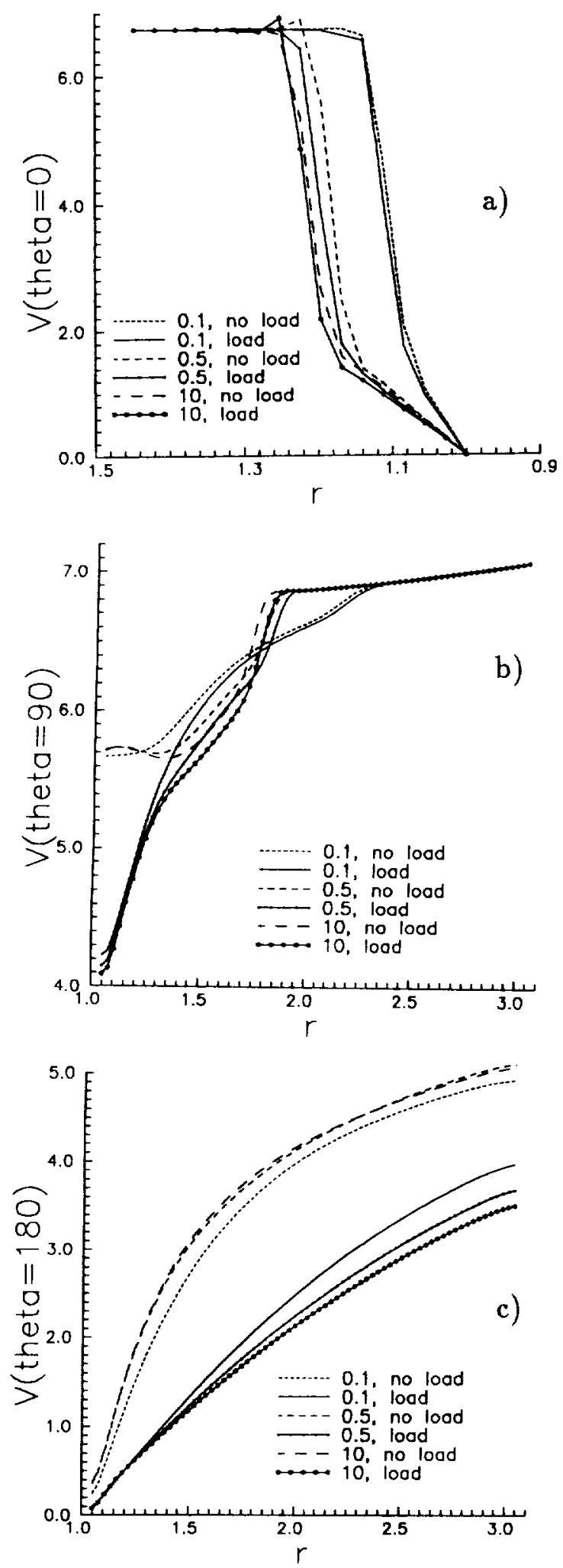

Figure 2. As in Figure 1 but for the normalized velocity profiles. very close to those presented in this paper. For the corresponding discussion, see also Cable and Steinolfson [1995].

The time step limit for methods using explicit temporal differencing for advective problems is the well-known
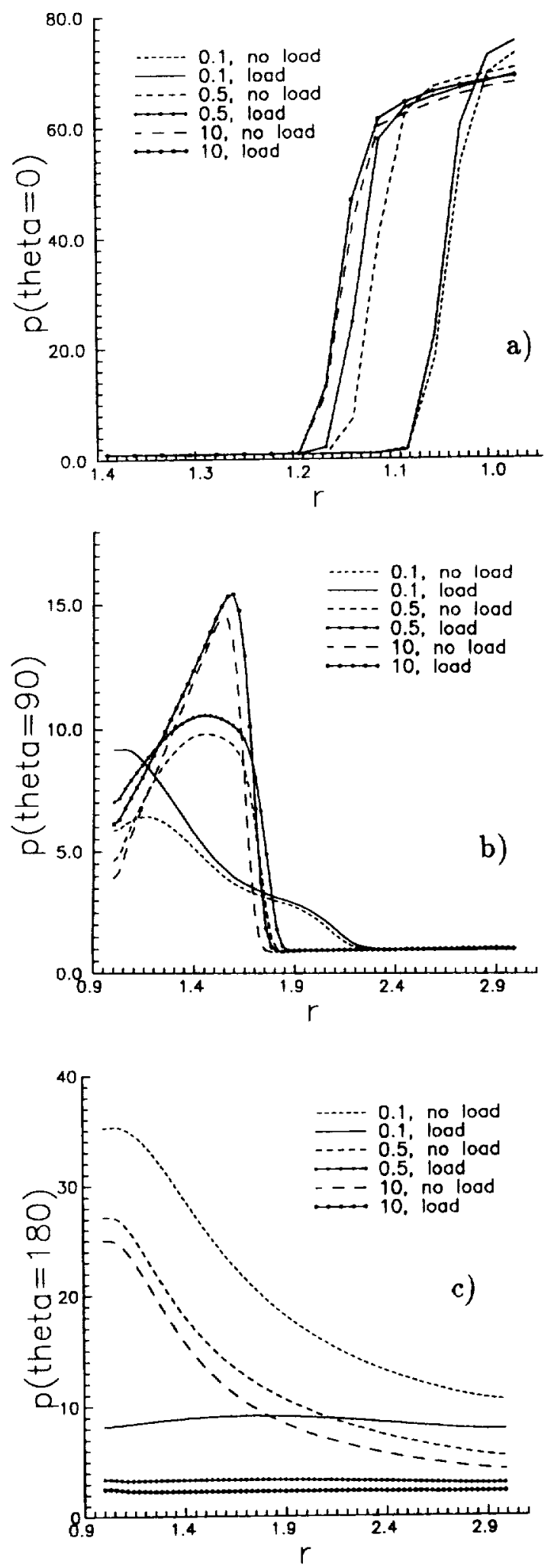

Figure 3. As in Figure 1 but for the normalized pressure profiles. 


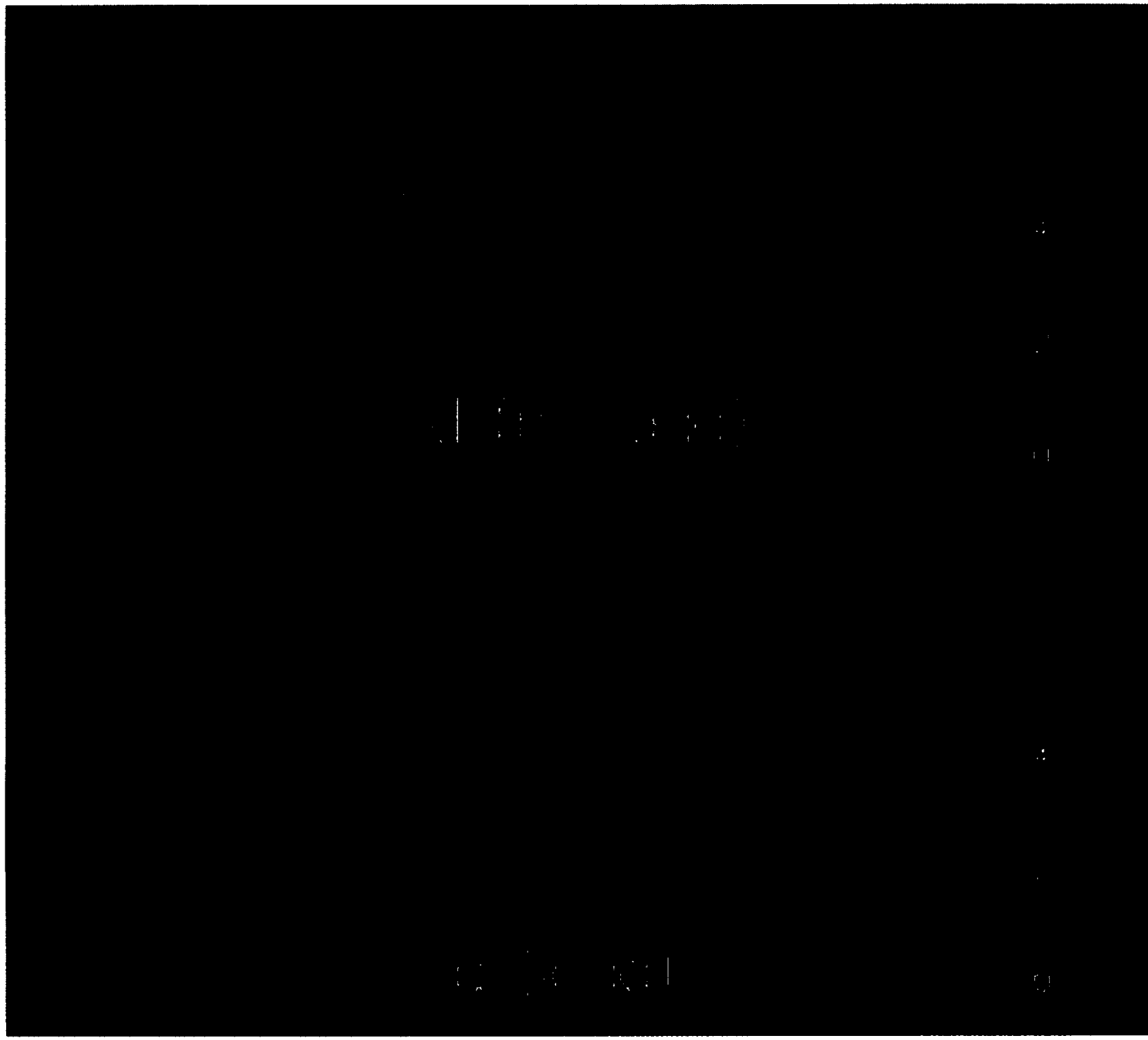

Plate 1. Normalized mass densities $\left(\rho / \rho_{\infty}\right)$ for the MHD non loaded (top) and mass loaded (bottom) simulations for the case of a parallel $(\mathbf{B} \| \mathbf{V})$ magnetic field and the plasma $\beta=0.5$. Here, $\rho_{\infty}$ is the density of the solar wind. The effect of mass loading (ML) is to increase the mass density in the overall region, except the tail region in which the mass density is reduced. $\rho_{\infty}$ denotes the solar wind mass density.

CFL condition, $\Delta t \leq \Delta l /|V|$, where the cell size is $\Delta l$ and $V$ is the fastest physical speed in the system. The resulting computer code [Cable and Steinolfson, 1995] is second-order accurate in space and time.

Defining a spherical boundary for the problem is easily accomplished. However, solving the equations of magnetohydrodynamics in spherical coordinates requires some modification of the usual Lax-Wendroff scheme. In particular, the singular points at $\theta=$ 0 and $\theta=180^{\circ}$ are handled through application of L'Hospital's rule to extrapolate values from grid points next to the singular points. Since the value of each variable at the singular points may vary depending on the angle $\phi$ at which the singular points are approached, a flux-weighted average of the values at all angles is used for the final updated value. This procedure is described in more detail by Cable and Steinolfson [1995].

We used a simple model for the boundary conditions that is physically motivated and performs adequately well. Venus is modeled as a hard, highly electrically conducting sphere. The inner boundary is taken at the planetary surface $\left(1 R_{v}\right)$ where the radial components of both the magnetic field and velocity are set to zero. All other physical variables are computed by extrapolation of values along a radial line. At the dayside of the outer radial boundary, all quantities are set to the solar wind values. The values at the nightside of the outer radial boundary are determined by extrapolation along the local flow direction. Moreover, we have never encountered transient conditions where the flow was coming in from the outer radial boundary. A typical computation begins with the introduction of the desired solar 
Plate 2. Normalized plasma flows $\left(V / a_{\infty}\right)$ for the MHD non loaded (top) and mass loaded (bottom) simulations for the case of a parallel $(\mathbf{B} \| \mathbf{V})$ magnetic field and the plasma $\beta=0.5$. Here, $a_{\infty}$ is the sound speed of the solar wind. The effect of ML is to decrease the plasma flow.

wind values in the dayside within the numerical box. The initial IMF is taken from the potential solution for flow over a sphere so that $\nabla \cdot \mathbf{B}=0$ in the initial state. The continued satisfaction of $\nabla \cdot \mathbf{B}=0$ is assured by solving a Poisson equation and updating the magnetic field. The numerical solution then continues until the interaction achieves an approximate steady state.

The density and pressure are initially constant through out the simulation region. The initial velocity field is the flow of an incompressible fluid over a sphere, directed along the $\theta=0$ line:

$$
\begin{gathered}
V_{r}(r, \theta, \phi, t=0)=-V_{0}\left[1-\left(\frac{R_{v}}{r}\right)^{3}\right] \cos \theta, \\
V_{\theta}(r, \theta, \phi, t=0)=V_{0}\left[1+\frac{1}{2}\left(\frac{R_{v}}{r}\right)^{3}\right] \sin \theta, \\
V_{\phi}(r, \theta, \phi, t=0)=0,
\end{gathered}
$$

where $V_{0}$ is the solar wind speed. The magnetic field is initialized in a similar way, except that $\mathbf{B}$ is skewed with respect to the solar wind flow by the desired angle. In the present paper, this angle is $0^{\circ}$. This is clearly not a frequently occurring case [Phillips et al., 1986], but it is a reasonable first-order approximation which will serve as a reference for comparison with results for cone angles of $45^{\circ}$ and $90^{\circ}$. These processes will be realistically modeled in future calculations.

\section{Results and Discussion}

We present all numerical results for the oxygen ion production rate $q_{0}=3 \times 10^{5} \mathrm{~cm}^{-3} \mathrm{~s}^{-1}$ [McGary and Pontius, 1994] and discuss several values of the plasma $\beta=8 \pi p_{0} / B_{0}^{2}$, namely $\beta=0.1, \beta=0.5, \beta=10$, and a typical value of $\beta=1.5$. The case of $\beta=0.1(\beta=10)$ corresponds to the strong (weak) magnetic field case. The physical solar wind values used are: electron density $n_{e}=20 \mathrm{~cm}^{-3}$, temperature $T=10^{5}{ }^{\circ} \mathrm{K}$, sound 


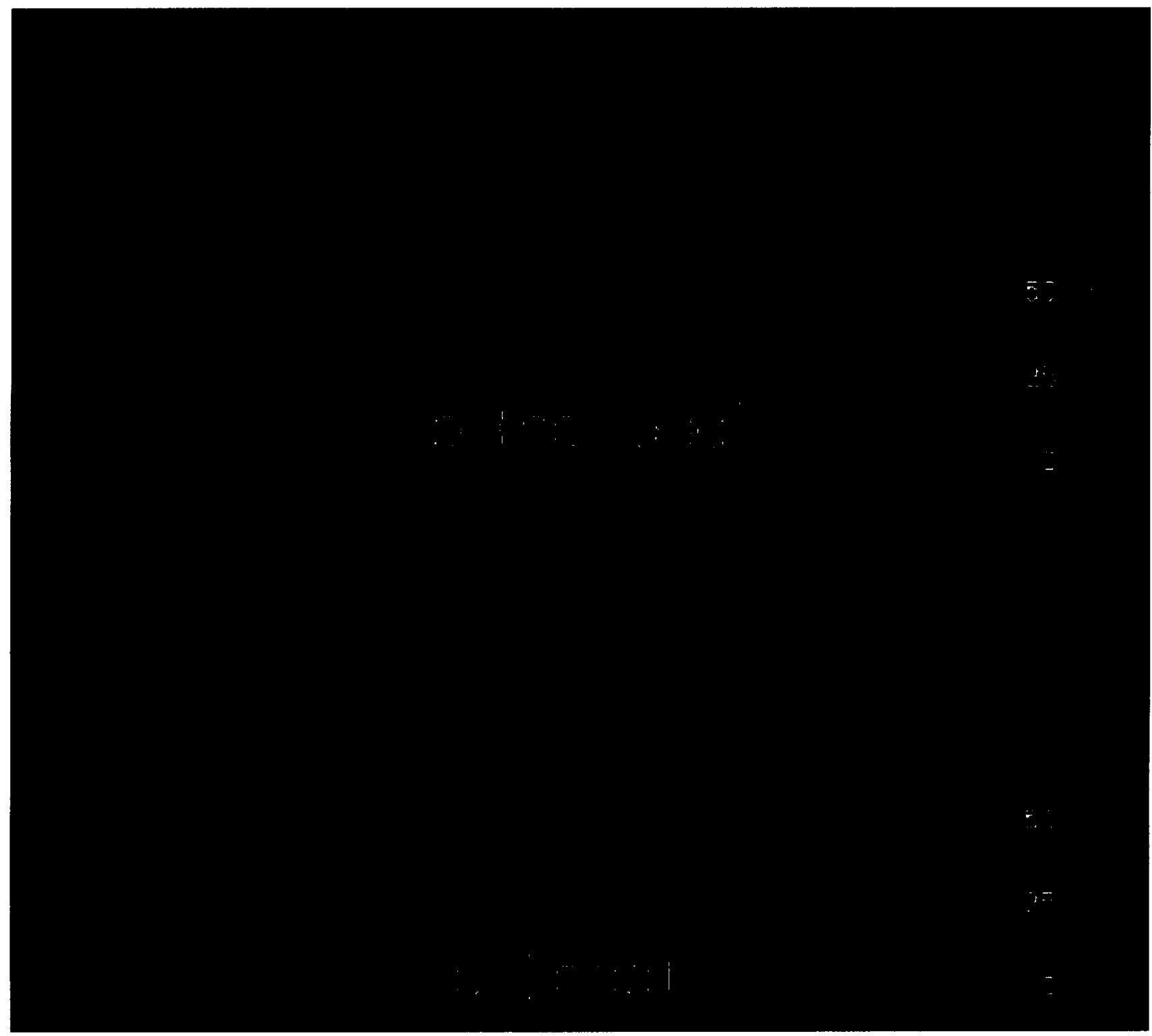

Plate 3. Normalized plasma pressure $\left(p /\left(\rho_{\infty} a_{\infty}^{2}\right)\right)$ for the MHD non loaded (top) and mass loaded (bottom) simulations for the case of a parallel (B\|V) magnetic field and the plasma $\beta=0.5$. The effect of $M L$ is to decrease the plasma pressure.

speed $55 \mathrm{~km} / \mathrm{s}$, flow velocity $370 \mathrm{~km} / \mathrm{s}$, sonic Mach number 6.73 , and thermal pressure $6.06 \cdot 10^{-10} \mathrm{dyn} / \mathrm{cm}^{2}$. Other physical parameters are chosen the same as in the work by Cable and Steinolfson [1995].

As a first step to apply the methodology to the problem of ML in the atmosphere of Venus, we consider an axisymmetric case with the magnetic field lines parallel to the solar wind flow. Consequently, $B$ is parallel to the Sun-Venus line and the entire solution is rotationally symmetric around this line, $\partial / \partial \phi=0$. We used the numerical technique described in the previous section to obtain a solution of the set of (1)-(5). The flow is from left to right and all physical quantities are either symmetric or antisymmetric about the lower boundary.

As shown in Plate 1, the mass density is increased in the case of mass loaded (ML) solutions. This finding is consistent with a simple analysis of mass continuity equation (1) and with previously published results [e.g., Linker et al., 1989; McGary and Pontius, 1994]. However, a more detailed close-up reveals that in the tail and terminator regions the mass density is reduced by the ML effects (Figure 1). Similar reduction is observed in the case of $\beta=\mathbf{0 . 5}$ along the Sun-Venus line, at $r \simeq 1.07 R_{v}$ (Figure 1a). The density reduction does not occur at the terminator for $\beta=0.1$, while this effect takes place for $\beta=0.5$ and $\beta=10$ (Figure $1 \mathrm{~b})$. The density depletion is stronger for a higher value of $\beta$. Just at the planetary surface, at $r<1.3$ $R_{v}$, the mass density increase is observed (Figure $1 \mathrm{~b}$ ). In the magnetotail, the plasma density reduction occurs at the planetary surface for all values of the plasma $\beta$. Again, the rate of density depletion is proportional to $\beta$. The case of $\beta=0.1$ differs from the other cases just as at the planetary surface $\rho$ is increased, but farther on there is a region of mass depletion which is followed by a region of mass enhancement (Figure 1c). To our 
Plate 4. Normalized plasma temperature $\left(T / T_{\infty}\right)$ for the MHD non loaded (top) and mass loaded (bottom) simulations for the case of a parallel (B\|V) magnetic field and the plasma $\beta=0.5$. The effect of $M L$ is to decrease the plasma temperature.

knowledge, this is the first report of mass depletion due to the ML. The density depletion is a consequence of the nonlinear term $\nabla \cdot(\rho \mathbf{V})$, which is smaller due to the plasma flow reduction by the ML effects. Indeed, the largest density (velocity) increase (decrease) occurs in the magentotail at $r=3 R_{v}$ for $\beta=0.1$. The normalized density jumps from $\rho_{N L} \simeq 1.25$ to $\rho_{M L} \simeq 1.5$ (Figure 1c), while the magnitude of plasma flow is reduced from $V_{N L} \simeq 4.9$ to $V_{M L} \simeq 4$ (Figure $3 \mathrm{c}$ ). Consequently, $\rho_{N L} V_{N L}=6.125>\rho_{M L} V_{M L}=6$. Therefore the effect associated with this nonlinear term overcomes the density increasing effect exerted by the exponential term $q_{0} \exp \left[-\left(h-h_{0}\right) / H_{0}\right]$. The density reduction in the tail may also be due to the changed shape of the bow shock. A different bow shock shape may result in less mass being carried into the tail, regardless of other factors.

It follows from Euler equation (2) that we can expect the reduction of a velocity magnitude because conservation of momentum and energy requires that the plasma flow is decelerated as a consequence of the mass density reduction. Plate 2 presents results both for the $\mathrm{ML}$ and no loading (NL) simulations. By examining the two solutions, one can see a fundamental difference between them. The effect of ML is to decrease the flow. A decreasing rate is highest in the tail region where slow flows in the case of NL are much more reduced by ML. The decreasing rate is larger for a smaller value of $\beta$ at the terminator and in the tail region. The plasma starts decelerating because of ML well ahead of the shock. In the subsolar region, this rate is largest for $\beta=0.5$. A large flow deceleration occurs also at the terminator, close to the planetary surface where the plasma flow drops from $V_{N L} \simeq 5.7$ to $V_{M L} \simeq 4.2$ (Figure $2 \mathrm{~b}$ ). Inspection of Plate 2 reveals the global structure of the shock and the stagnation region behind it. The flow 
decelerates at the shock as approaching solar wind absorbs an increasing number of ions. At the planetary surface, $r=R_{v}$, there is no perpendicular flow as a consequence of the boundary conditions imposed. Velocity shears in the radial direction are present at the bow shock region both for the ML and NL flows, which is
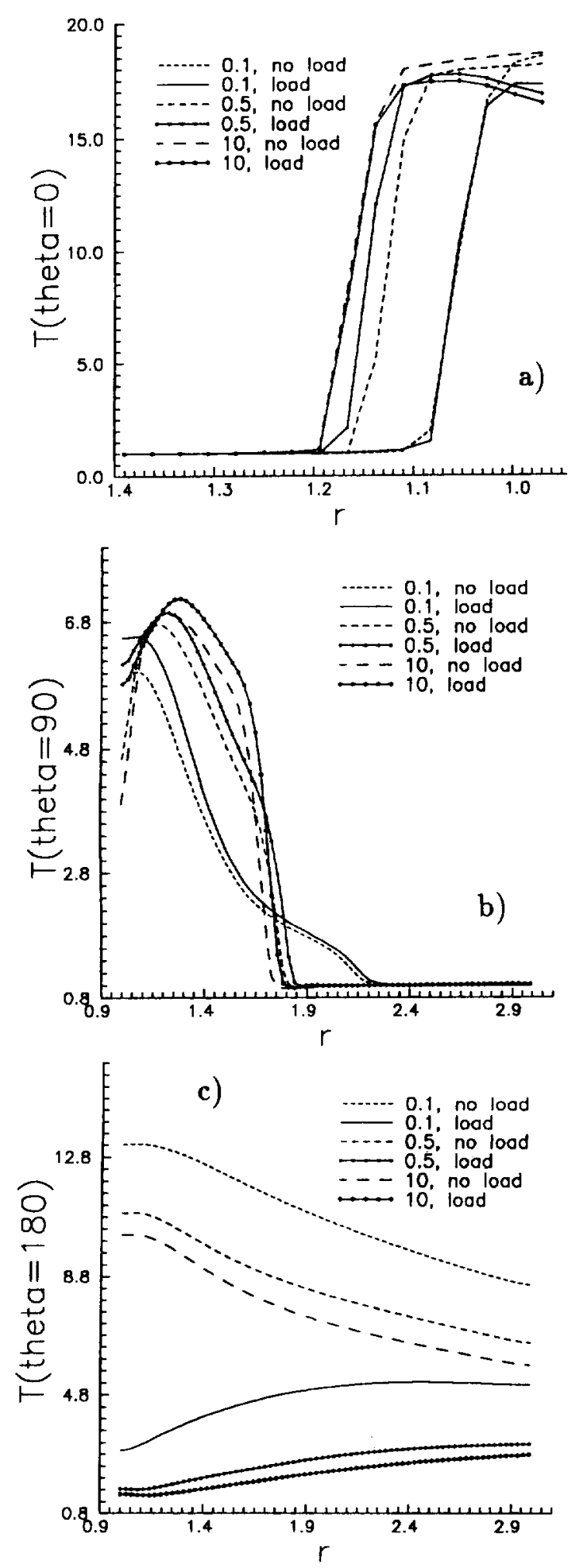

Figure 4. As in Figure 1 but for the normalized temperature profiles. consistent with PVO observations [Mihalov et al., 1982] and the gasdynamic results by McGary [1993].

From (4) we can derive an equation which governs the evolution of the NL plasma pressure $p$

$$
\frac{\partial p}{\partial t}+\nabla \cdot(p \mathbf{V})=(1-\gamma) p \nabla \cdot \mathbf{V}
$$
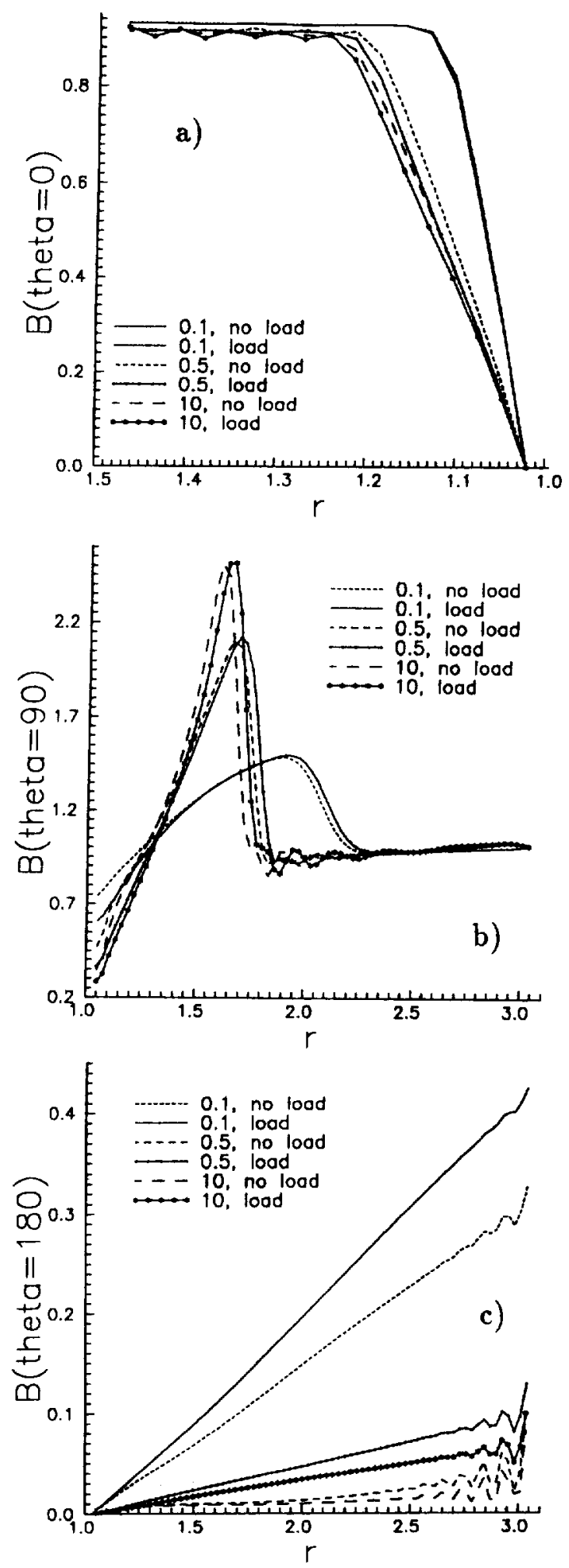

Figure 5. As in Figure 1 but for the normalized magnitude of the magnetic field profiles. 
As the plasma flow velocity $\mathbf{V}$ is decreased by the ML effects we can expect a pressure reduction. The simulation results shown in Plate 3 are qualitatively consistent with our expectations. However, Plate 3 shows that the plasma pressure is increased near the terminator region, in the region $\theta<90^{\circ}$. This increase is caused by a nonlinear interaction which is described by the $\nabla \cdot(p \mathbf{V})$ and $p \nabla \cdot \mathbf{V}$ terms. Our results differ from the conclusion made by McGary and Pontius [1994], who claimed that the dynamics of the plasma flow are not significantly affected by ML. A reason for the difference between the present results and those of McGary and Pontius has to do with the different models applied; McGary and Pontius performed gasdynamic (with $\mathbf{B}=\mathbf{0}$ ) simulations for the cylinder flow, whereas our model concerns MHD and a plasma sphere.

The above scenario may differ for different values of $\beta$. It occurs in the case of a sufficiently small value of $\beta$ that the magnetic field is so strong that the fast shock becomes an intermediate one [Steinolfson and Hundhausen, 1990]. For example, the ML increases the gas pressure along the whole Sun-Venus line in the case of $\beta=0.1$ and $\beta=10$ (Figure $3 \mathrm{a}$ ) but reduces it in the case of $\beta=0.5, \beta=1$, and $\beta=1.5$ at the planetary surface. In the bow shock region, an enhancement of $p$ due to the ML is observed for all values of $\beta$. The strongest enhancement seems to occur for $\beta=0.5$. At the terminator (Figure $3 \mathrm{~b}$ ), the gas pressure is increased by ML. The rate of decrease is highest at the planetary surface for $\beta=0.1$, while at larger distances the influence of ML on $p$ is negligible. The pressure reduction at the planetary surface is caused by the right-hand side term of (9) which becomes small at the surface because of the flow reduction. The bow shock is closer to the planetary surface for a higher value of $\beta$, in accordance with our expectations. At the magnetotail the pressure is reduced (Figure 3c). The reduction rate is highest at the planetary surface for the smallest value of $\beta$ and is a monotonic function of $\beta$.

The next point of comparison between the ML and NL results is the temperature distribution around Venus. As for the ideal gas the plasma temperature $T$ is proportional to $p / \rho$ so both $p$ and $\rho$ play a major role in determining its behavior. We already learned that for $\beta=0.5 \rho(p)$ is increased (decreased) by the ML effects everywhere except in the terminator and tail (nose) regions. Therefore, we may expect the general behavior of $T$ would be to decrease its magnitude by the $\mathrm{ML}$ effects. Indeed, Plate 4 indicates that the ML significantly cools the flow relative to the NL case. The cooling occurs as zero-temperature oxygen ions are introduced into the flow and thermalized [McGary and Pontius, 1994]. The behavior of $T$ is interesting to discuss in more detail as a function of the radial direction $r$ at different locations of $\theta$. In the case of $\theta=0$, for $\beta=0.1$ and $\beta=10$ the temperature is decreased by ML both at the planetary surface and at the sunward side of the bow shock (Figure 4a), while in the shock the effect of ML is negligible. The curves which correspond to the ML possess maxima in the magnetosheath, while no maxima exist for the NL case. A similar maximum occurs also in the

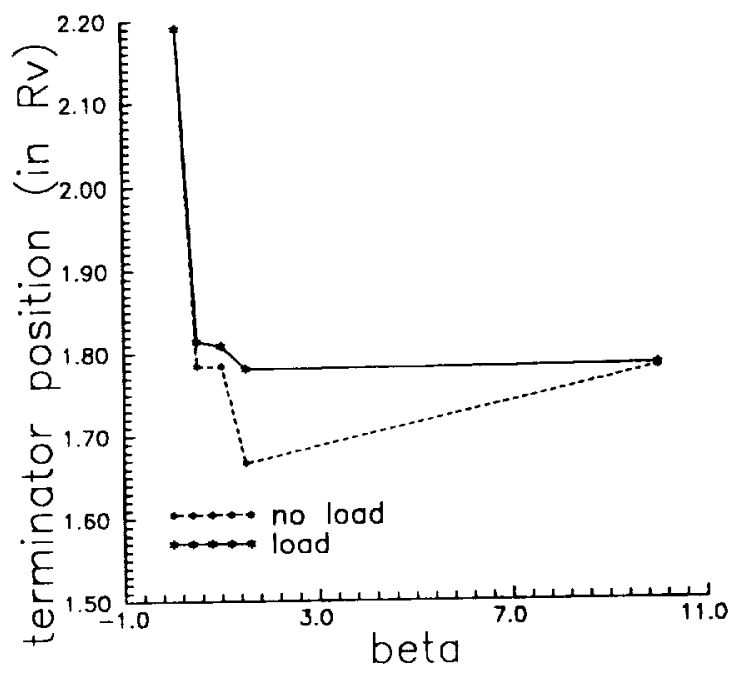

Figure 6. Terminator positions for different values of the plasma $\boldsymbol{\beta}$ and for the non loaded and mass loaded simulations. The bow shock at the terminator is shifter farther away farther away from the surface of Venus.

case of $\beta=0.5$ for the ML curve. Now, however, ML cools the plasma at the planetary surface but warms it in the bow shock region. The temperature increase at the bow shock was observed recently by Gombosi et al. [1994] in their simulations of the interaction of an expanding cometary atmosphere with the solar wind. At the terminator, the plasma is warmed by ML (Figure $4 b)$. The warming is more significant at the planetary surface and is higher for a higher value of $\beta$. The cooling occurs at the magnetotail (Figure $4 \mathrm{c}$ ). The cooling rate is higher at the surface of Venus and for a smaller value of $\beta$. This is a consequence of the pressure decrease at the planetary surface (Figure 3c). The mass density is increased for $\beta=0.1$ and decreased for $\beta=0.5$ and $\beta=10$, but the decreasing rate is smaller than the pressure decreasing rate (Figure 1c). Consequently, $T \sim p / \rho$ is much decreased at the surface of Venus. The cooling rate is much more significant than the warming effect. The bow shock position is closest to the planetary surface for the smallest presented values of $\beta$. The bow shock distance from Venus grows with $\beta$.

Plate 5 shows the magnitude of the magnetic field. In the subsolar region there is no jump in the magnitude of the magnetic field across the bow shock. At the flanks, however, the magnetic field increases through the bow shock. The magnetic tail is a region of reduced magnetic field. It can be seen that the ML exerts some influence on the magnetic field strength. Small differences occur at the sunward side of the shock where the magnetic field is decreased by the ML effects (Figure 5a). This effect is small for $\beta=0.1$ and grows with $\beta$. The decrease in the magnetic field strength nearest the planet along the stagnation streamline, is the result of the boundary conditions $\left(B_{r}\left(r=R_{V}, \theta, t\right)=0\right)$ imposed together with the fact that the parallel shock does not change a value of the magnetic field across the shock. As the planet is conducting there can be no radial magnetic field component at the surface of 
Venus. But near the subsolar line, the solar wind magnetic field is purely radial. Consequently, the magnetic field strength has to drop to zero in this region. This behavior counter to the common picture of $B$ piling up in the stagnation region in the case of $\mathbf{B} \perp \mathbf{V}$ [Cable and Steinolfson, 1995; Murawski and Steinolfson, 1995] and would only occur for the magnetic field nearly parallel to $\mathbf{V}$. At the terminator, the ML enhances the magnitude of $B$ at the bow shock. Therefore at the planetary surface $B$ is reduced. A decreasing rate is hardly dependent on $\beta$. The largest differences occur in the magnetotail where the magnetic field is enhanced by ML. The strongest enhancement occurs for $\beta=0.1$. This behavior contradicts our expectations, which follow a simple analysis of (3) wherein as a consequence of $V$ decrease we expect the magnetic field reduction by the ML effects. However, $\mathbf{V}$ couples nonlinearly with the magnetic field. The coupling occurs through the term $\nabla \times(\mathbf{V} \times \mathbf{B})$, which determines the overall behavior of the magnetic field rather than the plasma flow $\mathbf{V}$ only.

Inspection of Plates 1-5 reveals that a bow shock is formed upstream of Venus. The principal effects are that the bow shock is displaced farther from Venus in the case of the ML plasma [Belotserkovskii et al., 1987; Breus et al., 1989; McGary and Pontius, 1994]. For precise investigation of the bow shock structure, Figures 1-5 exhibit the plasma mass density, velocity, gas pressure, temperature, and magnetic field distribution along the Sun-Venus $\left(\theta=0^{\circ}\right)$, terminator $\left(\theta=90^{\circ}\right)$, and $\theta=180^{\circ}$ line. Inspection of these figures reveals that the pressure at the planetary surface is larger for a smaller value of $\beta$. The ML effects also shifts the shock at the terminator farther away from the planetary surface (Figure 6). This shift is largest for intermediate values of the plasma $\beta$. These results show the bow shock at the terminator is in about the position of the PVO observations [Tatrallyay et al., 1984; Russell et al., 1990]. Average subsolar and terminator distances of the bow shock are about $1.15 R_{v}$ and $2 R_{v}$ (from the center of the planet), respectively. To match the observed locations (at the nose) of the bow shock, the MHD model requires that the mass loading be greatly enhanced.

Russell and Zhang [1992] in their analysis of PVO data found that during periods when the solar wind magnetosonic Mach number is near unity and the plasma beta is low, the bow shock may travel to distances greater than $10 R_{v}$ away from the planet. On the basis of previous work on the formation of MHD shocks in coronal mass ejections [Steinolfson and Hundhausen, 1990], it was felt that the solar wind conditions had become (during the far bow shock excursions) such that the usual fast MHD shock is no longer a stable solution. The lower than normal plasma beta and magnetosonic Mach number are in a parameter regime for which the usual fast-mode bow shock close to the planet may not provide the necessary compression and deflection of the solar wind. Using MHD simulations, it was shown [Steinolfson and Cable, 1993] that, for these conditions, the usual fast shock is replaced by a bow shock

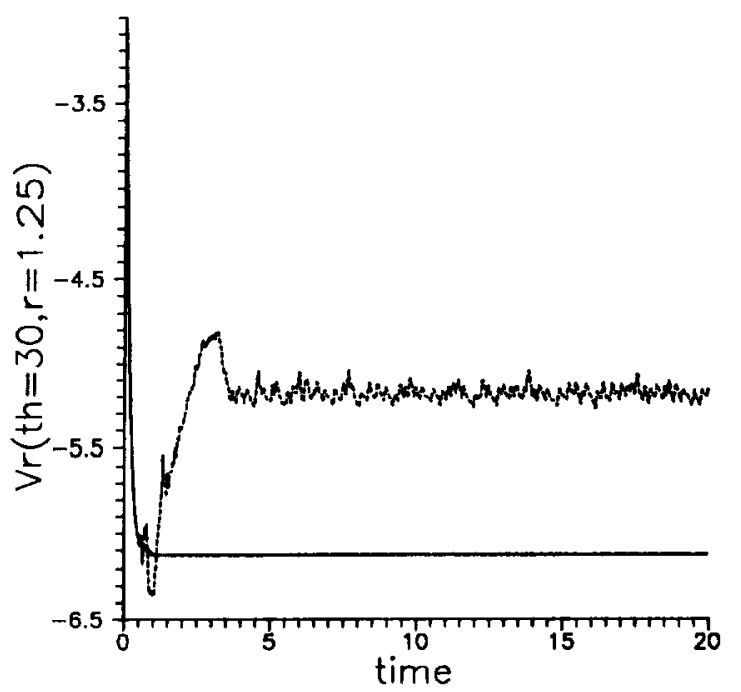

Figure 7. Time history of the radial velocity component $\left(V_{r} / a \infty\right)$ at the spatial position very close to the bow shock for the non loaded simulations. The upper (lower) curve corresponds to $\beta=$ $10(\beta=0.1)$. Not the stabilizing effect of the stronger magnetic field.

configuration containing an intermediate shock near the Sun-Venus line and a fast shock at large distances from the Sun-Venus line. The resulting configuration propagates upstream away from the planet at a low speed and appears to be approaching a new equilibrium stand-off location at a large distance from the planet.

Plates 1-5 exhibit waves which propagate along the bow shock boundaries. We have checked that these waves are not numerical artifacts as they do not disappear for a finer numerical grid and are persistent in time (not shown). These waves are generated by the solar wind impact, which is exerted by the solar wind on the plasma surrounding the planet. The first impact shifts the plasma towards the surface of Venus, while later in time the magnetic field recovers and the plasma is displaced farther away from the planet. Moreover, the bow shock first builds closer to the planet and then its altitudes at the equator $\left(\theta=0^{\circ}\right)$ and at the terminator $\left(\theta=90^{\circ}\right)$ grow in time. As a consequence, the nose of the bow shock oscillates in time. The Alfvén and slow oscillations, which are guided by the magnetic field lines [e.g., Murawski et al., 1995], are fed by the Kelvin-Helmholtz (KH) instabilities [e.g., Rankin et al., 1993], which are more apparent in the case of a weak magnetic field case. Figure 7 presents the radial component of the plasma flow as a function of time and for $\beta=0.1$ and $\beta=10$. This component is calculated at a spatial position which is very close to the bow shock. As expected, the stronger magnetic field makes the flow less unstable as the amplitude of oscillations is smaller in the case of $\beta=0.1$ than in the case of $\beta=10$. The KH instabilities criterion [e.g., Rankin et al., 1993]

$$
\omega_{i}^{2}=\frac{\rho_{1} \rho_{2}}{\left(\rho_{1}+\rho_{2}\right)^{2}}\left[\mathbf{k} \cdot\left(\mathbf{V}_{2}-\mathbf{V}_{\mathbf{1}}\right)\right]^{2}-\frac{\left(\mathbf{B}_{1} \cdot \mathbf{k}\right)^{2}+\left(\mathbf{B}_{\mathbf{2}} \cdot \mathbf{k}\right)^{2}}{4 \pi\left(\rho_{1}+\rho_{2}\right)}
$$


Plate 5. Normalized magnitude of the magnetic field $\left(B / \sqrt{\mu_{0} \rho_{\infty} a_{\infty}^{2}}\right)$ for the MHD non loaded (top) and mass loaded (bottom) simulations for the case of a parallel (B\|V) magnetic field and the plasma $\beta=0.5$. The effect of ML is to increase the magnitude of $B$ in the terminator region.

is satisfied easier for a less magnetized flow. In this formula indices 1 and 2 correspond to variables on different sides of the bow shock, while $\omega_{i}$ denotes the growth rate of the KH instabilities. It is easy to find out that the flow is unstable $\left(\omega_{i}^{2}>0\right)$ for a non-magnetic plasma $\left(\mathbf{B}_{1}=\mathbf{B}_{2}=0\right)$. On the other hand, strong magnetic fields can make the flow stable $\left(\omega_{i}^{2}<0\right)$. Intermediate values of the magnetic fields stabilize the flow and in particular the flow can be marginally stable $\left(\omega_{i}^{2}=0\right)$. These instabilities occur in the region where the flow has a large gradient such as at the bow shock. Assuming that the wave propagation $k$ is parallel to the flow $\mathbf{V}$ and magnetic field $\mathbf{B}$, it follows from (10) that

$$
\left(\rho_{1}+\rho_{2}\right)^{2} \frac{\omega_{i}^{2}}{k^{2}} / V_{A}^{2}=\rho_{1} \rho_{2}\left(V_{2}-V_{1}\right)^{2}-\left(\rho_{1}+\rho_{2}\right)\left(B_{1}^{2}+B_{2}^{2}\right),
$$

where now the plasma parameters are normalized by the units of the solar wind. $V_{A}$ is the Alfven speed of the solar wind. At the surface of Venus, $V_{2}=B_{2}=$ 0 . Upstream of the bow shock, the mass density $\rho_{1}$ and the plasma flow $V_{1}$ are hardly dependent on the plasma $\beta$. Therefore we can put $\rho_{1} \simeq 0.95$ and $V_{1} \simeq 6.7$ in normalized units. For $\beta=0.1(\beta=10)$ we have $\rho_{2} \simeq 3.9\left(\rho_{2} \simeq 3.7\right)$ and $B_{1} \simeq 0.93\left(B_{1} \simeq 0.91\right)$. It is easy to check that the right-hand side of $(11)$ is positive, indicating that the flow is $\mathrm{KH}$ unstable for both $\beta=0.1$ and $\beta=10$.

\section{Summary}

The interaction of the solar wind with Venus is studied using numerical solutions of the time-dependent ideal two - dimensional MHD equations. A modified Lax-Wendroft scheme [Cable and Steinolfson, 1995] is used to solve the equations in a spherical coordinate 
system. For these computations the detailed chemistry of the ionosphere is neglected, and the planet is treated as a conducting sphere. Numerical computations have been perfomed to study the effect of ML and the IMF strength on the magnetic barrier and the general configuration of the magnetic tail. The IMF orientation included in this study is parallel to the solar wind flow direction.

The main results are the following: The ML increases the mass density in the overall region, except the terminator and tail where the mass density depletion occurs as a consequence of the nonlinearity action. The mass density depletion is larger for a higher $\beta$. As a conseqence of this reduction, the plasma flow, pressure, and temperature are reduced. This scenario depends, however, on a value of the plasma $\beta$. It was found that for $\beta=0.1$ both the plasma pressure and the temperature are increased at the dayside by the ML.

Acknowledgments. The authors thank the referees and Jim Burch for their helpful comments on the earlier version of this paper. K. M. is grateful for Sam Cable's assistance on several numerical issues. This work was financially supported by NASA grant NAGW-4054 and by the Internal Research and Development Program at Southwest Research Institute.

The Editor thanks D. L. de Zeeuw and J. L. Phillips for their assistance in evaluating this paper.

\section{References}

Alexander, C. J., and C. T. Russell, Solar cycle dependence on the location of the Venus bow shock, Geophys. Res. Lett.,12, 369, 1985.

Belotserkovskii, O. M., T. K. Breus, A. M. Krymskii, V. Y. Mitnitskii, A. F. Nagy, and T. I. Gombosi, The effect of the hot oxygen corona on the interaction of the solar wind with Venus, J. Geophys. Res. Lett., 14, 503, 1987.

Biermann, L., B. Brosowski, and H. M. Schmitt, The interaction of the solar wind with a comet, Solar Phys., 1, 254, 1967.

Breus, T. K., S. J. Bauer, A. M. Krymskii, and V. Y. Mitnitskii, Mass loading in the solar wind interaction with Venus and Mars, J. Geophys. Res., 94, 2375, 1989.

Cable, S., and R. S. Steinolfson, Three dimensional MHD simulations of the interaction between Venus and the solar wind, J. Geophys. Res., 100, 21,645, 1995.

Cloutier, P. A., H. A. Taylor Jr., and J. E. McGary, Steady state flow/field model of solar wind interaction with Venus: Global implication of local effects, Planet. Space Sci.,22, $967,1987$.

Cravens, T. E., et. al., Disappearing ionospheres on the nightside of Venus, Icarus, 51, 271, 1982.

Gombosi, T. I., T. E. Cravens, A. F. Nagy, R. C. Elphic, and C. T. Russell, Solar wind absorption by Venus, J. Geophys. Res., 85, 7747, 1980.

Gombosi, T. I., K. G. Powell, and D. L. De Zeeuw, Axisymmetric modelling of cometary mass loading on an adaptively refined grid: MHD results, J. Geophys. Res.,99, 21,525, 1994.
Khurana, K. K., and M. G. Kivelson, A variable crosssection model of the bow shock of Venus, J. Geophys. Res., 99, 8505, 1994.

Linker, J. A., M. G. Kivelson, and R. J. Walker, The effect of mass loading on the temperature of a flowing plasma, Geophys. Res. Lett., 16, 763, 1989.

Luhmann, J. G., The solar wind interaction with Venus, Space Sci. Rev.,44, 241, 1986.

Luhmann, J. G., C. T. Russell, K. Schwingenschuh, and $Y$. Yeroshenko, A comparison of induced magnetotails of planetary bodies: Venus, Mars, and Titan, J. Geophys. Res.,96, 11,199, 1991.

McGary, J. E., Gasdynamic simulations of the solar wind interaction with Venus: boundary layer formation, Planet. Space Sci.,41, 395, 1993.

McGary, J. E., and D. H. Pontius Jr., MHD simulations of boundary layer formation along the dayside Venus ionopause due to mass loading, $J$. Geophys. Res., 99, 2289, 1994.

Mihalov, J. D., J. R. Spreiter, and S. S. Stahara, Comparison of gasdynamic model with steady solar wind flow around Venus, J. Geophys. Res.,87, 10,363, 1982.

Murawski, K., R. C. DeVore, S. Parhi, and M. Goossens, Numerical simulations of MHD wave excitation in bounded plasma slabs, Planet. Space Sci., in press, 1995.

Murawski, K., and R. S. Steinolfson, Numerical modelling of the solar wind interaction with Venus, Planet. Space Sci., in press, 1995.

Nagy, A. F., T. E. Cravens, J. H. Yee, and A. I. F. Stewart, Hot oxygen atoms in the upper atmosphere of Venus, Geophys. Res. Lett.,8, 629, 1981.

Phillips, J. L., J. G. Luhmann, and C. T. Russell, Magnetic configuration of the Venus magnetosheath, $J$. Geophys. Res., 91, 7931, 1986.

Phillips, J. L., J. G. Luhmann, C. T. Russell, and K. R. Moore, Finite Larmor radius effect on ion pickup at Venus, J. Geophys. Res., 92, 9920, 1987.

Rankin, R., B. G. Harrold, J. C. Samson, and P. Frycz, The nonlinear evolution of field line resonances in the Earth's magnetosphere, J. Geophys. Res., 98, 5839, 1993.

Rubin, E. L., and S. Z. Burstein, Difference methods for the inviscid and viscous equations of a compressible gas, $J$. Comput. Phys., 2, 178, 1967.

Russell, C. T., E. Chou, J. G. Luhmann, and L. H. Brace, Solar cycle variations in the neutral exosphere inferred from the location of the Venus bow shock, Adv. Space Res., 10, (5), 3, 1990.

Russell, C. T., and T. -L. Zhang, Unusually distant bow shock encounters at Venus, Geophys. Res. Lett.,19, 833, 1992.

Slavin, J. A., D. S. Intriligator, and E. J. Smith, Pioneer Venus Orbiter magnetic field and plasma observations in the Venus magnetotail, J. Geophys. Res., 94, 2383, 1989.

Spreiter, J. R., and S. S. Stahara, Solar wind flow past Venus: Theory and comparison, J. Geophys. Res., 85, $7715,1980$.

Spreiter, J. R., A. L. Summers, and A. Y. Alksne, Hydromagnetic flow around the magnetosphere, Planet. Space Sci., 14, 223, 1966.

Steinolfson, R. S., and S. Cable, Venus bow shocks at unusually large distances from the planet, J. Geophys. Res. Lett. 20. $755,1993$.

Steinolfson, R. S., and A. J. Hundhausen, MHD intermediate shocks in coronal mass ejections, J. Geophys. Res., 95, 6389,1990 .

Tanaka, T., Configurations of the solar wind flow and magnetic field around the planets with no magnetic field: Calculation by a new MHD simulation scheme, J. Geophys. Res., 98, 17,251, 1993.

Tatrallyay, M., C. T. Russell, J. G. Luhmann, A. Barnes, 
and J. D. Mihalov, On the proper Mach number and ratio of specific heats for modelling the Venus bow shock, J. Geophys. Res., 89, 7381, 1984.

Wu, C. C., MHD flow past an obstacle: Large-scale flow in the magnetosheath, Geophys. Res. Lett.,19, 87, 1992.

Zhang, T. L., J. G. Luhmann, and C. T. Russell, The solar cycle dependence on the location and shape of the Venus bow shock, J. Geophys. Res., 95, 14,961, 1990.

Zhang, T. L., J. G. Luhmann, and C. T. Russell, The magnetic barrier at Venus, J. Geophys. Res., 96, 11,145, 1991.
K. Murawski and R. S. Steinolfson, Department of Space Science, Southwest Research Institute, San Antonio, TX 782280510 (e-mail: KMurawski@solar.stanford.edu; richs@txdirect. net)

(Received March 16, 1995; revised August 3, 1995; accepted August 3, 1995.) 